\title{
VOLTAGE COLLAPSE MITIGATION BY REACTIVE POWER COMPENSATION AT THE LOAD SIDE
}

\author{
V Chayapathi ${ }^{1}$, Sharath $\mathbf{B}^{2}$, G S Anitha ${ }^{3}$ \\ ${ }^{1,2,3}$ Associate Professor, Electrical and Electronics Department, RVCE, Karnataka, India \\ chayapathiv@rvce.edu.in, sharathb@rvce.edu.in,anithags@rvce.edu.in
}

\begin{abstract}
Power system stability may be defined as the property of a power system that enables it to remain in a state of operating equilibrium under normal operating conditions and to regain an acceptable state of equilibrium after being subjected to a disturbance. A general and formal definition is given below. The IEEE/CIGRE Joint Task Force has proposed the following definition-"Power system stability is the ability of an electric power system, for a given initial operating condition, to regain a state of operating equilibrium after being subjected to a physical disturbance, with most system variables bounded, so that practically the entire system remains intact." The challenge faced in the modern power system is the collapse of voltages at heavy loads at the load buses of a power system. The voltage collapse will in turn will effect the stability of the power system. In this paper a novel way of mitigating the voltage collapse by supplying the reactive power at load buses is discussed and encouraging simulation results are obtained. The method employed is as fallows. Simulation is done without SVC and STATCOM at load buses. On comparison of the results at the load buses it is seen that with SVC and STATCOM at the load buses voltage collapse is mitigated in spite of heavily loading the system.
\end{abstract}

Keywords: Voltage Collapse, Reactive Power Management, Load Bus, Power System Stability.

\section{INTRODUCTION}

Stability in general, is a condition of equilibrium between opposing forces. In case of power systems, electric power generation and consumption are the opposing forces. Power system stability is the ability of the system to remain in operating equilibrium which is achieved between the electric power generation and consumption. Instability results when a system change or the disturbance leads to an imbalance between the forces in opposition. Power system stability can be classified as follows:

- Power system stability is classified into Rotor angle stability, Frequency stability and Voltage stability.

- The size of the disturbance: It can be a small scale disturbance (e.g. change in load) or a large disturbance (e.g. failure of a generator)

- The time span to be considered for assessing stability: Short term stability(of the order of several seconds) and Long term stability (of the order of several minutes)

Rotor angle stability refers to the ability of the synchronous machines of an interconnected power system to remain in synchronism after being subjected to a disturbance. It is further divided into small signal (or small disturbance) rotor angle stability and large signal rotor angle stability or transient stability. Frequency stability refers to the ability of the power system to maintain steady frequency following a severe disturbance, causing considerable imbalance between generation and load. Frequency stability can be a short term or a long term phenomenon. Voltage Stability Voltage stability is the ability of the power system to maintain steady acceptable voltages at all buses in the system at normal operating conditions and after being subjected to a disturbance. A power system is "voltage stable" if voltages at respective buses after a disturbance are close to voltages at normal operating conditions [1].

\section{VOLTAGE INSTABILITY AND VOLTAGE}

\section{COLLAPSE}

Voltage instability results from a progressive fall or rise of voltages of some buses. Voltage instability can be seen as uncontrollability of voltage magnitude at a number of buses of a power system.

Normally, power system operation is represented by power generation, load demand and adequate supply of power to loads at all times. Loads are usually the components which drive the power system to instability. When a disturbance occurs, the power consumed by loads is restored by the action of voltage regulators, tap changing transformers, etc., (load dynamics). These restored loads stress the high voltage network by consuming more reactive power. This further reduces voltages. The worst case situation arises when the above mentioned load dynamics increases the reactive power 
demand to such an extent that it exceeds the reactive power capability of the transmission and generation systems. Voltage instability is hence a state in which the power system no longer behaves in a predictable manner in response to operating changes. The following are the causes for voltage stability.

- Under load tap changing (ULTC) action during low voltage conditions: Several previous studies have shown that the conventional tap changer controller is an important contributor to voltage collapse, since it always aims to restore its controlled voltage, even following severe disturbances in the transmission system.

- Un favorable load characteristics[2]

- Poor co-ordination between various protective and control systems like generator excitation protection, armature over current protection, phase shifting regulators, etc.

- A small gradual system change such as normal increment in system load, leading to an initial voltage dip which in turn leads to further voltage dips.

\subsection{Voltage Collapse Mechanism}

A typical voltage collapse scenario is outlined below considering some types of system contingencies:

- Large generating units near the load centers are out of service and the power system experiences abnormal operating conditions. As a result, some EHV (Extra high voltage) lines are heavily loaded and reactive power resources are at a minimum.

- The loss of a heavily loaded line would cause additional loading on the remaining neighboring lines. Consequently, the reactive power losses in the lines would increase thereby resulting in heavy reactive power demand on the system.

- Immediately following the loss of the EHV line, a significant lowering of voltage at adjacent load centers would occur due to extra reactive power loading. However, the generator AVRs would quickly restore terminal voltages by increasing excitation. Increased voltage drop would occur in generators, transformers and lines due to additional reactive power flow through the inductances associated with these elements. At this stage, the armature and field current heating levels of generators would have reached their maximum limits.

- The decrease in EHV level voltage would be mirrored into the distribution system where ULTC substation transformers would take a few minutes ( 2 to 4 minutes) to restore distribution level voltages and loads to pre fault levels. Each step of this tap changing operation would result in incremental loading of EHV lines causing increased line losses which in turn would cause a greater drop in voltage levels. If this loading of the line exceeds the surge impedance loading, each further MVA increase in line flow would cause higher line losses.

\subsection{Prevention of Voltage Collapse}

This section discusses the several methods available for prevention of voltage collapse.

- Providing sufficient reactive power to a power system is the first defense against voltage collapse. Use of control systems such as fast-acting automatic voltage regulators, automatic controls for switching of capacitor banks. Use of Excitation control systems have to be properly set and co-ordinated. This is to ensure that the full reactive power capability of generators is available for system voltage support during decaying voltage conditions.

- Reducing Further the load is another means to provide support for system voltage. During decaying system voltage conditions, actions to increase distribution voltage levels by using tap changers can be blocked manually or automatically when collapsing voltage conditions are recognized.

- Load shedding programs are applied on a more common basis to prevent voltage collapse. Shedding load to mitigate voltage collapse should be implemented only after all other efforts to avoid a total voltage collapse have been exhausted. Manual load shedding programs can be effective in stabilizing a gradually collapsing system voltage. In such cases, pre-planned guidelines and procedures are developed for system operators to initiate load dropping when a voltage collapse is evolving. The difficulty with a manual system is that the burden is placed on human judgment to recognize the problem that exists and to determine that the only recourse is to shed load.

\subsection{Static VAr Compensator (SVC)}

Static VAr Compensator is a shunt connected static VAr generator or absorber whose output is adjusted to control current (capacitive or inductive) in order to maintain or control bus voltage magnitude.

SVCs are installed for many purposes such as

- Voltage regulation

- Reduction of voltage flicker caused by varying loads like arc furnace

- Increase power transfer capacity of transmission systems

- Increase transient stability limits of a power system

- Increase damping of power oscillations

- Reduce temporary over voltages

- Damp sub synchronous oscillations

The main advantage of SVCs over simple mechanicallyswitched compensation schemes is their near-instantaneous 
response to changes in the system voltage. For this reason they are often operated at close to their zero-point in order to maximize the reactive power correction they can rapidly provide when required. They are 1 cheaper, faster, and more reliable than dynamic compensation schemes such as synchronous condensers.

\subsection{Static Synchronous Compensator (STATCOM)}

It is based on a power electronics voltage-source converter and can act as either a source or a sink of reactive AC power to an electricity network. It is a member of the FACTS family of devices. Usually a STATCOM is installed to improve voltage stability. If the amplitude of output voltage is increased above the utility bus voltage, then a current flows from converter to ac system and the converter generates reactive power for the system. If the amplitude of the output voltage is decreased below the utility bus voltage, then the current flows from the ac system to the converter which absorbs the inductive reactive power from the ac system. If the output voltage equals the ac system voltage, reactive power exchange becomes zero and the STATCOM is said to be in floating state [3].

A STATCOM can improve power system performance in the following areas:

- Dynamic voltage control in transmission and distribution systems

- Power oscillation damping in power transmission systems

- Transient stability

- Voltage flicker control

- Control of active power in addition to reactive power in the connected line requiring dc energy source

\section{VOLTAGE STABILITY ANALYSIS}

The various methods available to study the voltage stability of a power system can be divided into static and dynamic analysis methods.

The static analysis provides snapshots of the system conditions at various instants of time and results in reducing the entire system equations to purely algebraic equations. In static analysis, the steady state stability of the system is determined using load flow equations. In these studies, it is assumed that all dynamics have died out and all controllers have performed their operations correctly. Steady state voltage stability studies pertain to long term voltage stability.

Dynamic analysis is based on the theory that voltage stability is a dynamic phenomenon. Time domain dynamic simulations enable representation of all possible incidents leading to voltage instability in chronological order. The dynamics of the power system is modeled as differential equations [4]. Dynamics of system's control and protection devices are considered so that practical and accurate definition of the system's stability limits is obtained more accurately.5]

\subsection{Static Voltage Stability Analysis}

The static approach captures snapshots of system conditions at various time frames along the time-domain trajectory. At each of these time instants, the time derivatives of the state variables are assumed to be zero, and the state variables take on values appropriate to the specific time frame. Consequently, the system can be represented in terms of purely algebraic equations allowing the use of static analysis techniques [6]

In the past, conventional power-flow programs using methods like Newton Raphson, Fast Decoupled Load Flow, etc., have been used for static analysis of voltage stability. In static voltage stability, slowly developing changes in the power system occur that eventually lead to a shortage of reactive power and declining voltage. This phenomenon can be observed by a plot of real power transferred versus receiving end voltage. This plot called PV curve was obtained at selected load buses earlier through power flows. PV curves can also be obtained as the output of continuation power flows or CPF by plotting voltage against the continuation parameter. The close relationship between reactive power and voltage allows another graphical representation of static voltage stability in the form of a plot between voltages at a bus versus the corresponding reactive power loading of the same bus. This graph can be plotted for different power factors and given values of real power and is called QV curve.

In addition to $\mathrm{PV}$ and $\mathrm{QV}$ curves, voltage stability indices have been formulated which serve as indicators of static voltage stability. These indices enable identification of weak parts of the system and also denote the closeness to voltage collapse.

\section{VOLTAGE STABILITY INDICES}

The purpose of voltage stability indices is to locate the weakest bus in the system and the most critical line connected to it. These indices may be based on the lines (Line voltage stability indices) or the bus (Nodal voltage stability indices)

- Line voltage stability Indices

1. Line stability index $\left(\mathrm{L}_{\mathrm{mn}}\right): \mathrm{L}_{\mathrm{mn}}=4 \mathrm{XQ}_{\mathrm{i}} /([\sin (\theta-$

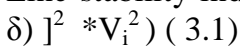

where $\mathrm{Q}_{\mathrm{i}}$ : Reactive powers at sending end bus

transmission line $\mathrm{X}=\mathrm{Z} \sin \theta, \mathrm{Z}$ is the impedance of the

$\theta$ : Power factor angle

$\delta$ : Voltage angle

$\mathrm{V}_{\mathrm{i}}$ : Sending end voltage

The system is stable if Lmn remains less than 1.

2. Line stability factor $\left(\mathrm{L}_{\mathrm{qp}}\right)$ : This index was proposed by A. Mohamed, et al. This index 
depends upon real power $\mathrm{P}_{\mathrm{i}}$ and reactive power $\mathrm{Q}_{\mathrm{i}}$ at the sending end bus.

$$
\mathrm{Lqp}=4\left(\mathrm{X} / \mathrm{V}_{\mathrm{i}}^{2}\right)\left(\left(\mathrm{X} / \mathrm{V}_{\mathrm{i}}^{2}\right) \mathrm{P}_{\mathrm{i}}{ }^{2}+\mathrm{Q}_{\mathrm{i}}\right)
$$

The system is stable if Lqp remains less than 1.

3. Fast Voltage Stability Index (FVSI): This index depends on the reactive power at the receiving end bus. This index is proposed by L Musirin Et.al. and is defined as

$\mathrm{FVSI}=4 \mathrm{Z}^{2} \mathrm{Q}_{\mathrm{j}} / \mathrm{V}_{\mathrm{i}}^{2} \mathrm{X}$

The system is stable if FVSI remains less than 1.

4. Voltage Collapse Proximity Index (VCPI): This index depends on the ratio of real power to maximum value of real power at the sending end and ratio of reactive power to maximum value of reactive power at the sending end. This index is proposed by M.Moghavemmi Et.al. and is defined as

VCPI $(P)=P j / P(\max )$

VCPI $(Q)=Q j / Q(\max )$

$\mathrm{Pj}$ and $\mathrm{Qj}$ are obtained from load flow analysis and $\mathrm{P}(\max )$ and $\mathrm{Q}(\max )$ are the maximum values of active and reactive powers which can be transferred through the line under consideration.

The system is stable if VCPI remains less than 1.

5. On Line Voltage Stability Index (LVSI)

An online voltage stability index is proposed from the view point of the relationships between line active power and bus voltage with the line. If the resistances of the transmission line are zero, then this index fails. The system is stable if LVSI is less than or equal to 1 .

$\mathrm{LVSI}=4 \mathrm{P}_{\mathrm{j}} \mathrm{R} /\left[\mathrm{V}_{\mathrm{i}} \cos (\theta-\delta)\right]^{2}$

- Nodal stability indices

1. L index: is a global indicator which provides quantitative estimate of distance of actual operating point of system to stability limit.

2. Diagonal element dependent index: An index based on partial derivatives of active and reactive powers with respect to voltage angle and voltage which indicates voltage stability.

This work focuses on use of line voltage stability indices in determining critical lines and hence the weakest bus in the power system [7].

\section{WSCC 9 BUS TEST SYSTEM}

This is a Western Systems Coordinating Council test system with three synchronous machines and nine buses. The synchronous machines are provided with IEEE type-1 exciters. The synchronous machines may be provided with automatic voltage regulators. The system consists of three loads at buses 5, 6 and 8 .

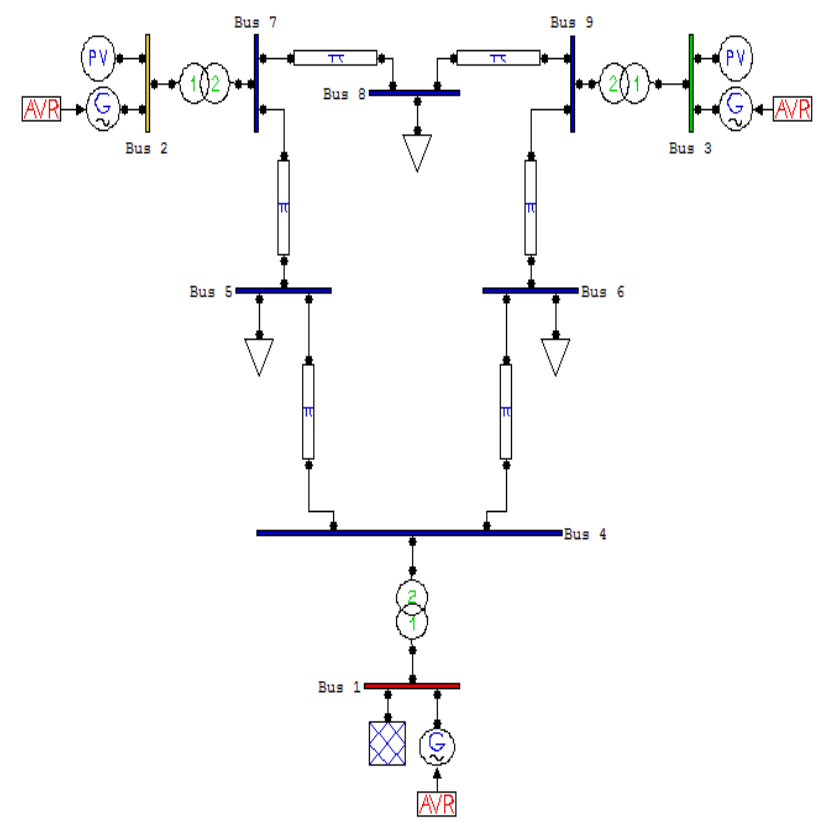

Fig 1 WSCC 9 BUS TEST SYSTEM

\subsection{Analysis of 9 Bus System Using Indices}

The 9 bus test system was analyzed on similar lines as the IEEE 14 bus test system. The system without any reactive power compensation by FACTS devices was considered and the load buses (5, 6 and 8 ) were subjected to various loadings. The loadings were of the form of increasing real power alone, increasing reactive power alone and then increasing both real and reactive powers simultaneously. The Newton Raphson power flow program was run for the various loadings and then the load flow results were entered into a MATLAB program which gave the Line Stability Index and Line Stability Factor as outputs. The critical lines were found to be lines 5 and 6 as indicated by the values of indices (closer to 1). These critical lines were found to be either directly connected to or in the proximity of bus 5 . Bus 5 was identified as the weakest bus and following connection of STATCOM at bus 5, the indices were recalculated.

\subsection{Analysis of 9 Bus System Using QV Curves}

QV curves were plotted for the critical bus 5. Bus 5 was made a PV bus by addition of a PV generator. Then, scheduling of voltage was done for bus 5 i.e. bus voltage was set at a particular value. Then the Newton Raphson power flow was run and the corresponding reactive power at bus 5 was obtained. This process was repeated with the voltage at bus 5 being incremented at the start of each load flow. The voltage and reactive power values were then used to obtain a plot of QV in Excel as shown in Fig 2 Then QV curve was obtained for the case of STATCOM being connected at bus 5 as shown in Fig 3 
Maximum reactive power for the case without compensation was $-3.17 \mathrm{pu}$ and with STATCOM it was $-3.35 \mathrm{pu}$ indicating that the connection of STATCOM improved the reactive power load ability and hence the voltage stability.

However, once again the QV curve for change in FACTS device to SVC or in position of connection of FACTS device rendered the same QV curve as shown in Fig 3.
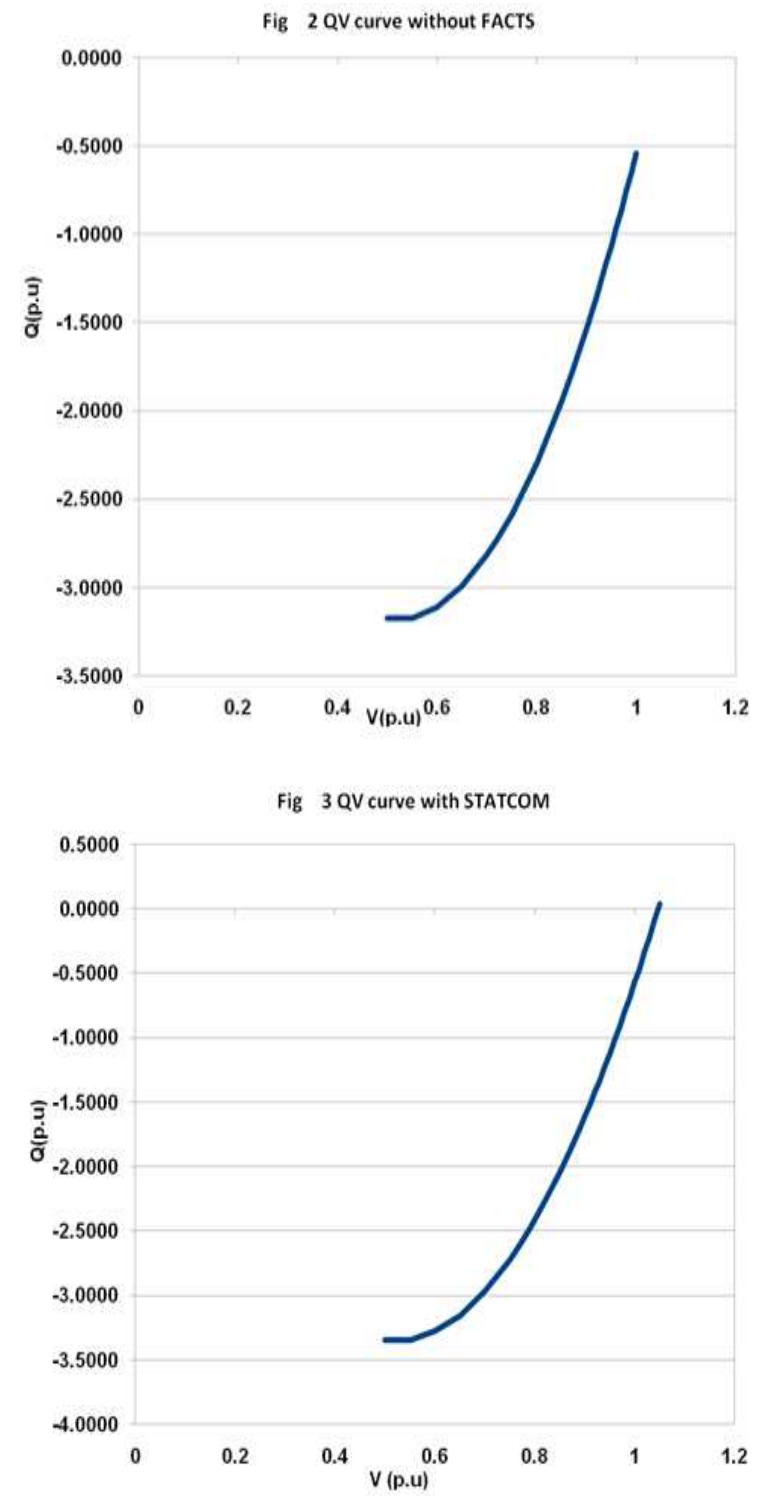

\subsection{Comparison of Sve/Statcom In 9 Bus System}

With Continuous Power Flow (Cpf)

The nine bus test system was subjected to continuation power flow and the maximum loading parameter was found to be 1.6216 .
SVC was first used to provide reactive power compensation. There were three possible locations for SVC, namely the load buses 5, 6 and 8. The best position for connection of SVC (Bus 8) was determined by running CPF after placing the SVC at the three load buses in turn. The PV curves in Fig 4 and the bar graph in Fig 5 illustrate that SVC at bus 8 provides the maximum load ability and hence improves voltage stability to the largest extent.

Next STATCOM was used for reactive power compensation and again the best place for its connection was determined. STATCOM was placed at the load buses of 5, 6 and 8 and the continuation power flow was run. Maximum lambda or loading parameter was obtained for STATCOM connection at bus 5 . Fig 6 and.7 show the PV curves and maximum loading parameter values for the three cases.

Finally three cases were taken and the continuation power voltage curves were obtained as shown in Fig 8. These cases were that of 9 bus system without FACTS, with SVC at bus 8 and with STATCOM at bus 5. As can be seen, SVC improved the system's real power load ability limit compared to the system with no reactive power compensation as can be seen from the "nose" of the curves. STATCOM further improved the voltage stability with the voltage showing little variation with real power loading when considered in the same range of loading as the SVC case. The bar graph in Fig 9 shows the values of maximum loading parameter.

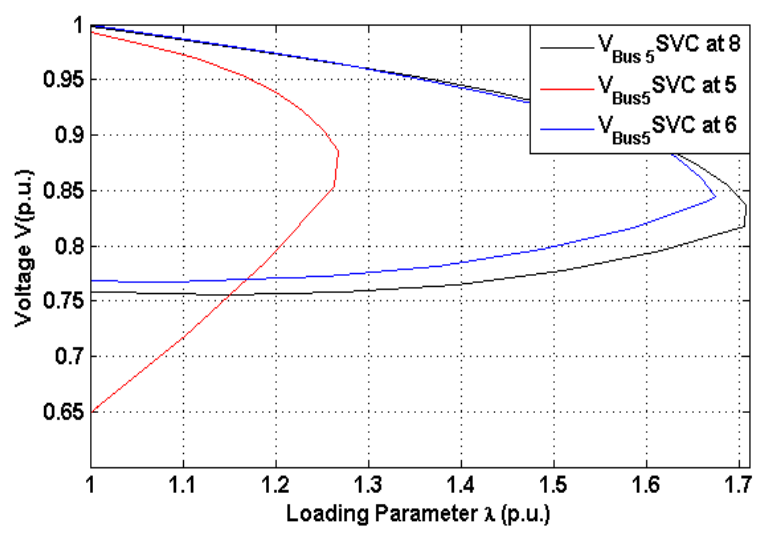

Fig 4 CPF curves for SVC 


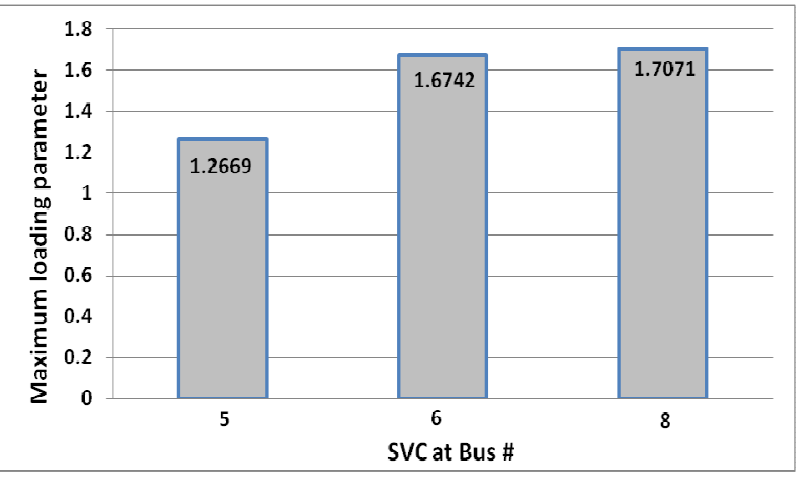

Fig. 5 Comparison of Lambda with different SVC positions

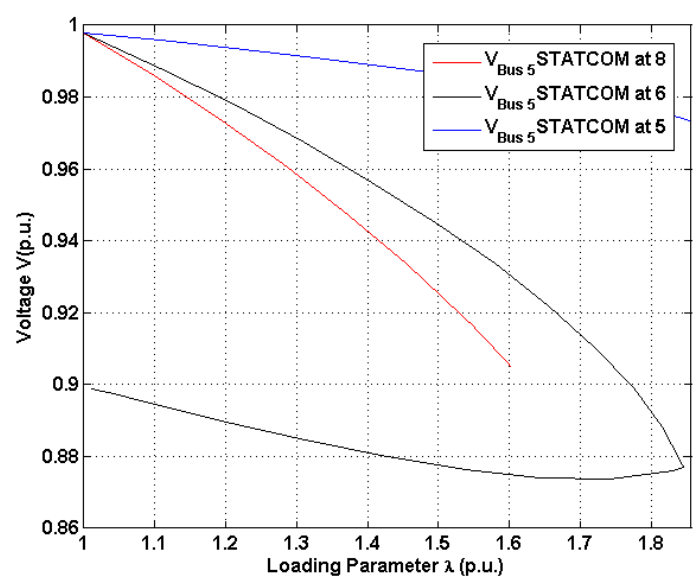

Fig. 6 CPF curves for STATCOM

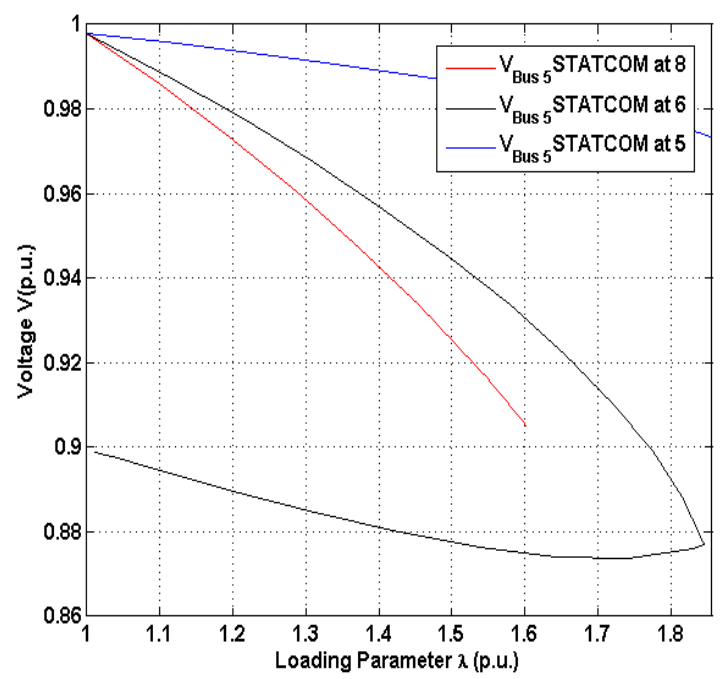

Fig 7 Comparison of Lambda with different STATCOM positions

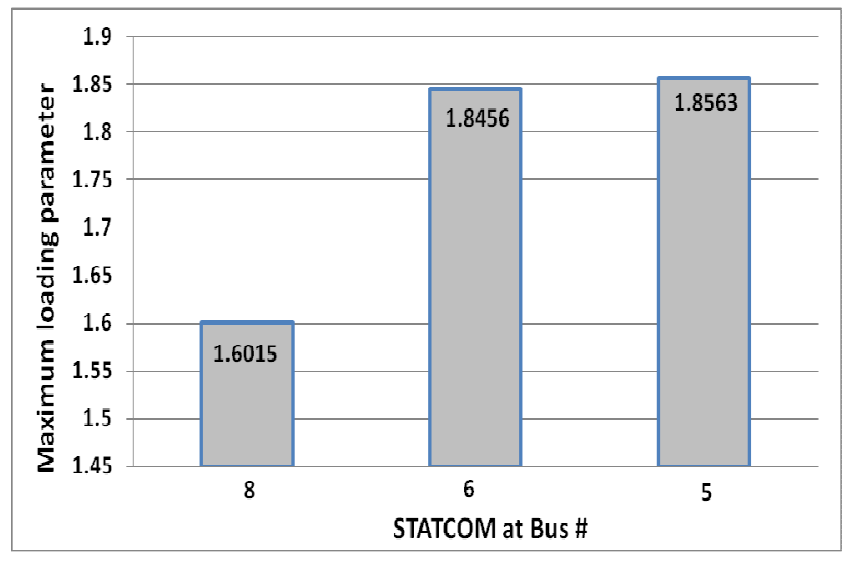

Fig 8 CPF Curves

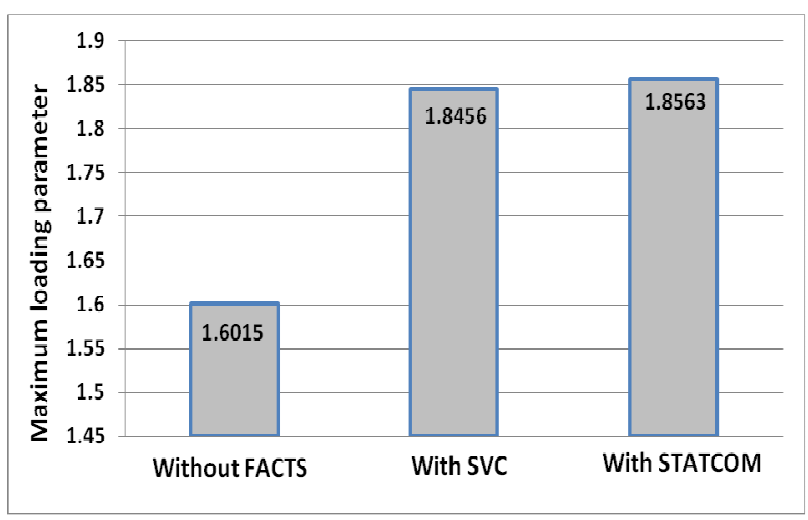

Fig .9 Comparison of lambda for different cases

\section{CONCLUSIONS}

The required objective is fulfilled.The results show that with FACTS devices the power system is less prone to voltage collapse at the load buses during heavily loaded conditions on the load buses. The reactive power supplied at the load buses has improved the power system stability.

\section{REFERENCES}

[1] Voltage Regulation with STATCOMs: Modeling, Control and Results- Amit Jain, Karan Joshi, Aman Behal, Ned Mohan, IEEE TRANSACTIONS ON POWER DELIVERY, VOL. 21, NO. 2, APRIL 2006.

[2] Improvement of STATCOM Performance with Optimum LQR and Pole placement Controller Based on Genetic Algorithm-S.Eshtehardiha, Gh.Shahgholian. First Joint Congress on Fuzzy and Intelligent Systems, Ferdowsi University of Mashhad, Iran

[3] Single phase statcom -its control algorithm-Linju Jose

[4] Analysis and Operation of STATCOM in Unbalanced Systems Carlos A.C. Cavaliere, Edson H. Watanabe , Maurício Aredes for StatCom Controls Operation with Unbalanced Voltages, Clark Hochgraf \& Robert H. 
Lasseter, Electrical and Computer Engineering Department, University of Wisconsin-Madison, Madison, WI 53706

[5] S. Mori, K. Matsuno, M. Takeda and M. Seto, "Development of a Large Static VAR Generator Using Self-Commutated Inverter For Improving Power System Stability," IEEE Trans. Power Systems, Vol. 8, No. 1.

[6] N. G. Hingorani, L. Gyugyi, "Understanding FACTS Concepts and Technology of Flexible AC Transmission Systems", IEEE Press, New York, 1999.

[7] Yves Narcisse Nguegan Tchokonte, Real Time Identification and Monitoring of Voltage Stability Margin in Electrical Power Transmission Systems Using Synchronised phasor measurements, Kassel University Press GmbH

[8] Venkataramana Ajjarapu, Computational Techniques for Voltage Stability Assessment and Control, Springer, 2006

[9] J.Lewis Blackburn and Thomas J. Domin, Protective Relaying: Principles and Applications, CRC Press, 3rd Edition, 2006

\section{BIOGRAPHIES}

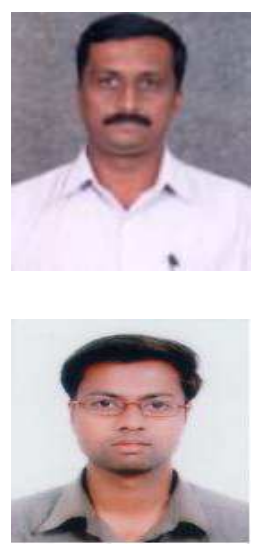

Prof. V. Chayapathy is working as an Associate Professor in the Dept of Electrical and Electronics, R V College of Engineering Bangalore. His areas of interest are Voltage Collapse, Power Electronic system design using IC's, Software systems.

Sharath B is working as an Assistant Professor in the Dept of Electrical and Electronics, R V College of Engineering Bangalore. His areas of interest are Renewable Energy Sources, Power Electronics \& Drivers, Image Processing

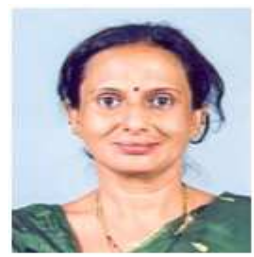

Dr G S Anitha is working as an Associate Professor in the Dept of Electrical and Electronics, R V College of Engineering Bangalore. Her areas of interest are Renewable Energy Sources, High Voltage Engineering 